\title{
Acknowledgement to Reviewers of Journal of Marine Science and Engineering in 2019
}

\author{
JMSE Editorial Office \\ MDPI, St. Alban-Anlage 66, 4052 Basel, Switzerland \\ Published: 16 January 2020
}

The editorial team greatly appreciates the reviewers who have dedicated their considerable time and expertise to the journal's rigorous editorial process over the past 12 months, regardless of whether the papers are finally published or not. In 2019, a total of 476 papers were published in the journal, with a median time to first decision of 17 days and a median time from submission to publication of 41 days. The editors would like to express their sincere gratitude to the following reviewers for their generous contribution in 2019:

$\begin{array}{ll}\text { Abdel-Hamid, Ayman } & \text { Almeida, Marisa } \\ \text { Abdelkhalik, Ossama } & \text { Alobaid, Falah } \\ \text { Abdussamie, Nagi } & \text { Alongi, Daniel M. } \\ \text { Abramović, Borna } & \text { Alsadik, Bashar } \\ \text { Acanfora, Maria } & \text { Alsweiss, Suleiman } \\ \text { Acharya, Tri Dev } & \text { Altosole, Marco } \\ \text { Acomi, Nicoleta } & \text { Alves, Tiago } \\ \text { Adam, Frank } & \text { Amaechi, Chiemela Victor } \\ \text { Adam, George } & \text { Amillis, Sotiris } \\ \text { Adams, Aaron } & \text { Amin, Mohammad Asif } \\ \text { Adegboye, Mutiu Adesina } & \text { An, Jinwoo } \\ \text { Aderinto, Tunde } & \text { Anavatti, Sreenatha G. } \\ \text { Afonso, Clélia N. } & \text { Ancellin, Matthieu } \\ \text { Afonso, Fernando } & \text { Anderlini, Enrico } \\ \text { Aggrey, John } & \text { Anderson, Eric } \\ \text { Akan, Cigdem } & \text { Anderson, Eric R. } \\ \text { Akbar, Muhammad K. } & \text { Anderson, Stuart } \\ \text { Al Mohit, Md. Abdul } & \text { Ando, Keita } \\ \text { Albatal, Ali } & \text { Andreetta, Anna } \\ \text { Alberello, Alberto } & \text { Andriolo, Umberto } \\ \text { Alcaide, Juan Ignacio } & \text { Anfuso, Giorgio } \\ \text { Al-Dahidi, Sameer } & \text { Angelidis, Dionysios } \\ \text { Aleksandrov, Mitko } & \text { Ansarifard, Nazanin } \\ \text { Alessandra, Romolo } & \text { Antonini, Alessandro } \\ \text { Alexandrakis, George } & \text { Antonio Augusto, Sepp Neves } \\ \text { Alin, Bosioc } & \text { Antunes Do Carmo, José Simão } \\ \text { Aliyu, Aliyu } & \text { Antunes, Carlos M. Correia } \\ \text { Alizad, Karim } & \text { Arai, Ryuta } \\ \text { Aljinović, Dunja } & \text { Arcas, Diego } \\ \text { Allahdadi, Mohammad Nabi } & \text { Archetti, Renata } \\ \text { Almeida Ferreira, Hugo } & \text { Arima, Masakazu } \\ \text { Almeida, Luis Pedro } & \text { Aristodemo, Francesco } \\ \text { A } & \end{array}$


Armstrong, Roy A.

Askarinejad, Amin

Atanasoae, Pavel

Austin, Martin

Aversa, Stefano

Ayca, Aykut

Ayed, Samah Ben

Azamathulla, Hazi M

Bacosa, Hernando P.

Bahrami, Shahab

Bai, Fushi

Bai, Wei

Baldauf, Michael

Baldi, Franceso

Balduzzi, Giuseppe

Bałdysz, Zofia

Balitsky, Philip

Ballesta, Monica

Baltazar, João

Baltzer, Agnès

Banerjee, Amit

Bangga, Galih

Baquerizo, Asunción

Barbaro, Giuseppe

Barbieri, Maurizio

Barltrop, Nigel

Barnard, Patrick

Barrineau, Patrick

Barsi, Dario

Basu-Gupta, Sudeshna

Beegle-Krause, Cynthia Juyne

Behrendt, Cezary

Belgiorno, Giacomo

Belibassakis, Kostas

Bell, Paul

Belotti, Giorgio

Benaouicha, Mustapha

Benbouzid, Mohamed

Benelli, Sara

Beniuga, Razvan

Benoit, Michel

Benshila, Rachid

Bergillos, Rafael J.

Bernard, Guillaume

Bertoni, Duccio

Besio, Giovanni

Beyabanaki, S. Amir Reza

Bhalla, Amneet

Bhattacharya, Subhamoy

Bhowmik, Basuraj

Bhushan, Shanti

Bialik, Robert
Billard, Jean Yves

Bilskie, Matt

Biolchi, Sara

Bishoge, Obadia

Biswas, Wahidul K.

Blanco, Juan

Blanco, Pilar

Boes, Robert

Bohle, Martin

Bokuniewicz, Henry J.

Bolbot, Victor

Bolles, André

Bonin-Font, Francisco

Bonomo, Anthony L.

Borkowski, Piotr

Borquist, Eric

Borthwick, Alistair

Bos, Arthur R.

Bosa, Silvia

Boufadel, Michel

Bouhmala, Noureddine

Branco, Paulo

Brandini, Carlo

Brandner, Paul A.

Branger, Hubert

Breddermann, Karsten

Brennan, Anthony B.

Briganti, Riccardo

Briggs, Tiffany

Brocchini, Maurizio

Brodie, Kate

Brus, Jan

Brycki, Bogumil

Bucci, Vittorio

Buccino, Mariano

Bucur, Daniel

Burdziakowski, Pawel

Burgdorf, Martin

Burian, Adina

Bustamante, Eliseo

Bustos, Alejandro

Caggiano, Antonio

Cagnoli, Bruno

Calabrese, Luigi

Calcagni, Danilo

Calisal, Sander

Camiz, Sergio

Campbell, Anthony

Cândido, José J.

Candries, Maxim

Cannata, Giovanni

Cantero Chinchilla, Francisco Nicolás 
Cao, Gui-ying

Capello, Marco

Capra, Alessandro

Čarija, Zoran

Carlos José, González

Carrillo Sánchez, José María

Carrington, Emily

Casalino, Damiano

Casavola, Alessandro

Caseiro Rocha, Alfredo Moreira

Cavagnaro, Robert

Cavallaro, Antonio

Cavallaro, Luca

Cave, Rachel

Cefalo, Raffaela

Celli, Daniele

Cely, Juan S.

Ceppi, Claudia

Cerik, Burak Can

Cerra, Bruno

Chabchoub, Amin

Chadli, Mohammed

Chang, Chih-Hua

Chang, Ching-Chih

Chang, Yeon S.

Charykova, Marina V.

Chau, Kwok-Wing

Chekurov, Sergei

Chen, Bang-Fuh

Chen, $\mathrm{Ru}$

Chen, Tai-Cheng

Chen, Wei-Bo

Chen, Yang

Cheng, Jie

Cheng, Zhengshun

Cheong, Cheolung

Cherubini, Claudia

Chiavola, Ornella

Chiu, Linus Y.S.

Cho, Hyun Jung

Choi, Hojong

Choi, Jungho

Chou, Ming-Tao

Christodoulou, Anastasia

Chu Van, Thuy

Chu, Philip

Church, Ian

Chyau, Charng-Cherng

Chybowski, Leszek Marek

Clain, Stephane

Clausen, Johan

Clavero, María
Cohn, Nicholas

Coleman, Shirley

Collins, Keri

Collu, Maurizio

Conides, Alexis

Conley, Daniel

Contardo, Stephanie

Contestabile, Pasquale

Contreras-López, Manuel

Convertino, Matteo

Coppola, Luigi

Corbí, Hugo

Correia, Sérgio

Cosoli, Simone

Cossu, Piero

Costas, Susana

Covelli, Carmine

Cowan, Zara-Louise

Cox, Dan

Cox, Ron

Cox, Rónadh

Craciun, Eduard-Marius

Craiger, J. Philip

Cruz-Romero, Pedro

Csilla, Stenger-Kovács

Cucinotta, Filippo

Cui, Fangda

Curto, Domenico

Czermański, Ernest

Czymzik, Markus

Dahl, Peter

Daidzic, Nihad

Dalla Valle, Giacomo

Damasevicius, Robertas

Damiani, Leonardo

Daniel, Priour

Darani, Shadi

Das, Anup

Davidson, Josh

Davidson-Arnott, Robin

Davies, Ian

Dawid, Leszek

De Bestern, Henk

De Carolis, Giacomo

De Castro Rodríguez, $\mathrm{M}^{\mathrm{a}}$ Teresa

De Luca, Pierantonio

De Sanjosé Blasco, José Juan

De Serio, Francesca

Degiuli, Nastia

Degtyarev, Alexander

Delefortrie, Guillaume

Delfs, Jens-Olaf 
Demirbilek, Zeki

Denamiel, Cléa

Denissenko, Petr

Deniziak, Stanisław

Dennington, Simon

Dev, Arun

Devolder, Brecht

Dhaliwal, Gurpinder

Dhanak, Manhar R.

Dharmarathne, Suranga

Di Bona, Gianpaolo

Di Ciaccio, Fabiana

Di Francesco, Silvia

Di Luccio, Diana

Di Noia, Luigi Pio

Di Risio, Marcello

Di Vaio, Assunta

Diamond, Howard J.

Díaz-Hernández, Gabriel

Dietz, Marianne

Dimas, Athanassios

Djordjevic, Nenad

Do, Jong-Dae

Dolores Molero, Gemma

Dombek, Grzegorz

Donnarumma, Silvia

Doong, Dong-Jiing

Dornbusch, Uwe

Dos Santos, Elizaldo Domingues

Drimer, Nitai

Drissi-Habti, Monssef

Drygała, Izabela Joanna

Dubbioso, Giulio

Dudojc, Boleslaw

Dulebenets, Maxim A.

Duplančić Leder, Tea

Duque, Carlos

Dussin, Raphael

Dysarz, Tomasz

Earthy, Jonathan

Edge, Billy

Edokpayi, Joshua Nosa

Edwards, Brandon

Eiksund, Gudmund Reidar

Eitzinger, Christian

El Moctar, Bettar O.

Elibol, Armagan

Eliopoulou, Eleftheria

El-Naas, Muftah H.

Elorza, Iker

Elsaesser, Bjoern

Elsafti, Hisham
Elsaid, Khaled

El-Shafee, Omar Osama

Emery, Brian

Esnaola, Ganix

Esteban, Maria Dolores

Etemaddar, Mahmoud

Eugenio, Francisco

Eun, Jongwan

Everett, Mark

Evers, Frederic

Eyal, Gal

Fabbri, Paolo

Fabrizio, Pistani

Faiña, Andres

Fairley, Iain

Faizi, Koohyar

Falahat, Saeed

Falco, Pierpaolo

Falkowska, Ewa

Faraci, Carla

Feil, Roland

Feldman, Daniel

Fernandez, Alberto

Fernandez-Gamiz, Unai

Fernandez-Garcia, Javier

Ferradosa, Tiago

Ferrando, Ilaria

Ferrari, Francesco

Ferrera, Maxime

Ferris, Timothy

Fiedler, Julia

Figlus, Jens

Figlus, Tomasz

Figueira, William

Fingas, Merv

Firth, Callum R.

Fischer, Andrew

Fischer, Jeoffrey

Fitzgerald, Colm

Fleming, Alan

Fonseca, Rita Maria Ferreira

Fornaser, Alberto

Forristall, George Z.

Forte, Giovanni

Fortuna, Luigi

Foster-Martinez, Madeline

Foumani, Mehdi

Fournier, Georges

Foysi, Holger

Fragassa, Cristiano

Frangeš, Stanislav

Frangopoulos, Christos 
Frankovska, Jana

Franzitta, Vincenzo

Frédéric, Muttin

Free, Andrew

Freedman, Jeff

Fricke, Wolfgang

Frigard, Peter

Fröhle, Peter

$\mathrm{Fu}$, Weiqi

Fuentes-Yaco, César

Fujimura, Atsushi

Fulford, Richard S.

Gabl, Roman

Gaeta, Maria Gabriella

Gallerano, Francesco

Ganske, Anette

Gao, Meng

García Espinosa, Julio

García, Juan T.

García-de-Lomas, Juan

García-gracia, Miguel

Garner, Andra J.

Garrote Revilla, Julio

Gauden, Piotr A.

Gawdzińska, Katarzyna

Gedikli, Ersegun Deniz

Geiger, Gerhard

Gelabert, Bernadí

Gentemann, Chelle L.

George, Douglas A.

Gervino, Gianpiero P.

Ghahremani, Kasra

Ghaisas, Niranjan

Ghofrani, Hadi

Ghommam, Jawhar

Ghori, Muhammad Usman

Ghosh, Samrat

Giannone, Francesca

Giardino, Alessio

Gilja, Gordon

Gioia, Andrea

Gioia, Dario

Giorgi, Giuseppe

Giorgio, Diana

Girton, James

Glaser, Sebastien

Gligor, Adrian

Głuchowski, Andrzej

Glynn, Peter J.

Goldstein, Evan B.

Golias, Mihalis

Gollasch, Stephan
Golub, Mikhail V.

Gomez Chavez, Arturo

Gómez, Fernando

Gomez-Pujol, Lluis

Gomiero, Alessio

Gomiz-Pascual, Juan Jesús

González, Aridane González

González, Jose

González-Gorbeña, Eduardo

González-Villanueva, Rita

Goodrich, Gregory B.

Goran, Bakalar

Gorman, Andrew

Goulas, Athanasios

Goward Brown, Alice

Grabowski, Martha

Granberry, Phillip J.

Gray, Tim

Grech, Michelle Rita

Greenslade, Penelope

Griffen, Blaine D.

Grigoropoulos, Gregory

Grimshaw, Roger

Groothuis, Peter

Grządziel, Jarosław

Grządziela, Andrzej

Guajardo, Mario

Gualtieri, Carlo

Gualtieri, Paola

Guan, Pai-Chen

Gucma, Lucjan

Gucma, Maciej

Guglielmino, Eugenio

Guyondet, Thomas

Haces-Fernandez, Francisco

Hahn, Axel

Hale, Rip

Haller, Merrick

Hally, David

Hamed, Ali

Hann, Christopher

Hänninen, Maria

Hansen, Anders Hedegaard

Harris, Larry

Hart, Patrick

Haugen, Stein

Havukainen, Jouni

Hayes, Donald

Hazelwood, Richard A.

Hekkenberg, Robert

Helfer, Véronique

Hemmati, Arman 
Hendrickson, Kelli

Henesey, Lawrence E.

Hequette, Arnaud

Herdzik, Jerzy

Herget, Jürgen

Herman, Przemyslaw

Herrero, Elías Revestido

Herterich, James

Hesham El, Askary

Hetzel, Yasha

Heunecke, Otto

Higuchi, Tomihiko

Hill, Jon

Hillis, Andrew J.

Hiraishi, Tetsuya

Hirdaris, Spyros

Hisaki, Yukiharu

Hlaváčová, Irena M.

Ho, Lanh Si

Hoecker-Martinez, Martin

Hole, Lars Robert

Honegger, David

Hong, SeungHo

Hong, Sup

Honkonen, Tuula

Horozal, Senay

Hossain, Md. Delwar

Hosseinzadeh, Mehdi

Hoth, Julian

Hovem, Jens

Hsin, Ching-Yeh

Hsu, Hungchu

$\mathrm{Hu}$, Howard $\mathrm{H}$.

Huang, Danwei

Huang, Hsin-Haou

Huang, Tse-Hung

Huang, Wei Po

Huang, Weimin

Hübler, Clemens

Hughes, Andrew

Hungerford, James

Huynh, Thanh-Canh

Hwang, Chi-Hung

Ibarra-Berastegi, Gabriel

Ichikawa, Kaoru

Iglesias, Gregorio

Imai, Yasutaka

Imani, Mahdi

Inazu, Daisuke

Ingrassia, Michela

Inhestern, Lukas Benjamin

Iris, Cagatay
Islam, Mohammad

Istrati, Denis

Ito, Junshi

Itsukushima, Rei

Iuppa, Claudio

Ivanov, Alexey V.

Jafarishiadeh, Mahdi

Jaffe, Jules

Jaime, Arriaga

Jajac, Nikša

Jaskólski, Krzysztof

Jastrzębska, Małgorzata

Jaworski, Justin

Je „drasik, Jan

Jeffrey, Robert

Jehel, Pierre

Jeng, Dong-Sheng

Jeong, Eui Seob

Jeong, Inbae

Jeong, Jungsik

Jeung, In-Seuck

Ji, Jian

Jia, Boru

Jiménez, José

Jin, Yuting

Johnson, Erick

Johnson, Markes

Johnstone, Cameron

Jolivet, Aurelie

Joshi, Sanjeev

Ju, Shen-haw

Jung, Jung-Yeul

Jurca, Florin

Justino, Paulo

Kabiersch, Grit

Kagemoto, Hiroshi

Kalaitzidis, Stavros

Kalra, Tarandeep S.

Kämpf, Jochen

Kantamaneni, Komali

Kanzaki, Yoshiki

Kapustin, Ivan A.

Kara, Kursat

Karabil, Sitar

Karahalios, Hristos

Karambas, Theophanis V.

Karbalaei Baba, Alireza

Karki, Sita

Karymbalis, Efthimios

Kasiulis, Egidijus

Kastrisios, Christos

Kasumyan, Alexander 
Käyhkö, Jukka

Kazemi, Amirkhosro

Kelpšaitè-Rimkienè, Loreta

Keramaris, Evangelos

Kerpen, Nils B.

Keshtpoor, Mohammad

Kessler, G.C.

Khanna, Shruti

Khatibi, Akbar Afaghi

Kianejad, S.S.

Kiang, Jean-Fu

Kim, Youngho

Kim, Yun-Tae

Kinnas, Spyros A.

Kiraga, Marta

Kishore, Ravi Anant

Kitsiou, Dimitra

Klarin, Branko

Klonaris, Georgios

Kobayashi, Kazumichi

Koch, Reinhard

Kodikara, Jayantha

Kokou, Fotini

Kolander, Robert

Kolerski, Tomasz

Kolomenskiy, Dmitry

Kolymbas, Dimitrios

Komba, Shiro

Kombiadou, Katerina

Konlechner, Teresa M.

KONNO, Yoshihiro

Konovessis, Dimitrios

Konstantinidis, Efstathios

Kontes, Georgios D.

Kordella, Stavroula

Kornev, Nikolai

Kos, Serdjo

Koubogiannis, Dimitrios

Koukouvinis, Foivos (Phoevos)

Kovacevic, Meho Sasa

Kovács, János

Kowalczewska-Madura, Katarzyna

Kozak, Anna

Kozłowski, Andrzej

Krata, Przemyslaw

Kress, Nurit

Krieger, Michael

Křivý, Vit

Krvavica, Nino

$\mathrm{Ku}$, Cheng-Yu

Kudlek, Edyta

Kumavor, Patrick D.
Kurkina, Oxana E.

Kurowska, Krystyna

Kuwahara, Takuya

Kwan, Wing Shun

Łabuz, Tomasz

Lacal Arantegui, Roberto

Lader, Pål Furset

Laface, Valentina

Lamas Galdo, María Isabel

Lambert, Martin

Landmann, Jannis

Lane, Emily

Lane-Serff, Gregory

Lashley, Christopher $\mathrm{H}$.

Laureano-Rosario, Abdiel E.

Laurens, Jean Marc

Lauria, Agostino

Lavrova, Olga

Lawrence, Peter

Lazarowska, Agnieszka

Le Tissier, Martin

Lebailly, Philippe

Lebeau, Juliana

Leccis, Francesca

Lee, Chun-Woo

Lee, Derher

Lee, Doo Young

Lee, Eunsu

Lee, Sang Bong

Lehr, Christian

Lei, Po-Ruey (Barry)

Lemu, Hirpa G.

Lengani, Davide

Lepidi, Marco

Levacher, Daniel

Levy, Jason K.

Lewis, Matthew

Li, Chunyan

Li, Hua

Li, Ji-Hong

Li, Linyan

Li, Yang

Li, Yuxing

Liao, Chien-Sen

Liberzon, Dan

Libric, Lovorka

Licitra, Gaetano

Ligęza, Paweł

Ligteringen, Han

Lim, Jun Heok

Lim, Seunghoo

Lim, Youngsub 
Lin, Aqiang

Lin, Chih-Hong

Lin, Hanzhi

Lin, Lee-Yaw

Lindstad, Elizabeth

Lira, Cristina

Lira-Loarca, Andrea

Lisitsyna, Liubov S.

Lisowski, Edward

Litwin, Wojciech

Liu, Shaoqing

Liu, Yonggang

Liu, Yucheng

Liversage, Kiran

Llorente Isidro, Miguel

Lo Presti, Diego

Lo Re, Carlo

Lomonaco, Pedro

Loncar, Goran

Longo, Sandro

López Ruiz, Alejandro

Loukogeorgaki, Eva

Lucia, Umberto

Ludeno, Giovanni

Lui, Eric

Luigi, Mucerino

Lund-Hansen, Lars Chresten

Lungu, Adrian

Luo, Min

Lusito, Letizia

Luttenberger, Axel

Maccarrone, Vincenzo

Macfarlane, Gregor

Madurapperuma, Buddhika

Makinson, Keith

Maklakov, D.V.

Makris, Christos

Malara, Giovanni

Malcangio, Daniela

Mali, Matilda

Malvic, Tomislov

Mancini, Simone

Manenti, Sauro

Manfrida, Giampaolo

Manno, Giorgio

Manzetti, Sergio Otterlei

Marcel·la, Castells

Marence, Miroslav

Margui, Eva

Márialigeti, Károly

Mariani, Guido Stefano

Marieu, Vincent
Marino, Enzo

Marino, Raffaele

Marsalek, Blahos

Martinelli, Luca

Martìnez Monterrubio, Sergio Mauricio

Martínez, Emilio Pascual

Martinez-Mora, Juan Antonio

Martins, Marcos Silva

Mason-Jones, Allan

Mata, Maria Del Carmen

Matias, Sessarego

Matic, Frano

Matono, Paula

Matsuda, Yoshitaka

Matsumoto, Hironori

Mattei, Cesar

Mattei, Gaia

Matulic, Maja

Matys Grygar, Tomas

Mauro, Francesco

Mautner, Andreas

Mazarakos, Thomas P.

Mazzola, Silvia Michela

McCarroll, Jak

McCay, Deborah French

Mcgookin, Euan

McGuffin-Cawley, James

Mehbub, Mohammad Ferdous

Meisina, Claudia

Memos, Constantine D.

Menza, Charles

Mereuta, Elena

Mewis, Peter

Miah, Abdul Halim

Michailides, Constantine

Michalopoulou, Zoi Heleni

Mikkelsen, Ove

Miller, Ian

Minea, Alina Adriana

Miot Da Silva, Graziela

Miramontes, Elda

Mishra, Anand

Mityagina, Marina I.

Miura, Takeshi

Miyata, Naoyuki

Mizutani, Norimi

Molfetta, Matteo Gianluca

Molines, Jorge

Molyneux, David

Monieta, Jan

Moñino, Antonio

Montessori, Andrea 
Montewka, Jakub

Montoya, Luis

Morago, Brittany

Morales González, Juan Antonio

Morea, Donato

Mori, Masaaki

Moro, Lorenzo

Morri, Carla

Mortazavi, Amir

Mos, Benjamin

Mottola, Fabio

Moura Paredes, Guilherme

Mousaviraad, Maysam

Mrzljak, Vedran

Mulvaney, Robert

Murphy, Jimmy

Murray, Harry

Muskett, Reginald

Mustafa, M. Aral

Musumeci, Rosaria Ester

Muttin, Frédéric

Nag, Soumyadeep

Nanni, Loris

Nash, Roisin

Nazarov, Andrej P.

Neagoe, Mircea

Neary, Vincent

Neely, Merrie

Neppalli, Venkata Kishore

Neumann, Philipp

Newell, Carter R.

Nicholas, William Anthony

Nikolic, Mijo

Nisheva, Maria

Nishi, Yoshiki

Nivedita, Nivedita

Noblesse, Francis

Nobre Silva, Ana

Noguchi, Tamao

Nordam, Tor

Nordhagen, Håkon

Nozawa, Yoko

Ntouskos, Valsamis

Nunes, Leonel

Nuzzo, Genoveffa

O'Loughlin, Conleth D.

Oberle, Ferdinand K.J.

Odetti, Angelo

Oleinik, Phelype Haron

Olivares, Lucio

Omer, Joshua

Onas, Adrian S.
Onea, Florin

Ordonez Sanchez, Stephanie

Orsini, Amandine

Orszaghova, Jana

Ortega, José Marcos

Ortiz-Suslow, David

Osinenko, Pavel

Osório, Jonas $\mathrm{H}$

Ostadhassan, Mehdi

Otis, Daniel

Otremba, Zbigniew

Ouahsine, Abdellatif

Ovalle, Carlos

Ozgokman, Tamay

Padrón, Luis A.

Palacios, Filiberto

Paldor, Nathan

Palmese, Maria

Pan, Yang

Papadakis, George

Papadimitriou, Kimon

Papadopoulos, Nikos

Papaspyrou, Sokratis

Parilkova, Jana

Park, Sang Kil

Park, Youngsoo

Parlagreco, Luca

Parsegov, Sergei

Parunov, Josko

Pasanisi, Francesco

Pascal, Rémy Claude René

Pascolo, Sara

Pasquali, Davide

Pasquero, Claudia

Pauer, James

Pawson, David

Pazouki, Kayvan

Pelinovsky, Efim

Pellegrinelli, Alberto

Pempkowiak, Janusz

Peñalba, Markel

Pennetta, Micla

Pensieri, Sara

Pepi, Milva

Pérennou, M. André

Pérez Arribas, Francisco

Pérez Rojas, Luis

Pérez-Alberti, Augusto

Perez-Collazo, Carlos

Pervizpour, Mesut

Petersen, Mark

Petrich, Jan 
Petti, Marco

Pfost, Heiner

Phillips, Andrew J.K.

Phillips, Steve

Piano, Marco

Pienkowski, Krzysztof

Pilecki, Zenon

Pinault, Jean-Louis

Piroddi, Luca

Pizarro, Alonso

Płaczek, Bartłomiej

Plesiński, Karol

Pochwat, Kamil

Poguluri, Sunny Kumar

Politis, Gerasimos

Postacchini, Matteo

Potts, Donald C.

Pourret, Olivier

Prendergast, Luke J.

Prezioso, Maria

Price, Timothy

Pritz, Balazs

Prpic-Orsic, Jasna

Pucher, Johannes

Pugliese Carratelli, Eugenio

Pullano, Salvatore

Pummer, Elena

Puzdrowska, Marta

Quaranta, Franco

Race, Marco

Radulovic, Jovana

Raileanu, Alina Beatrice

Rakowska, Aleksandra

Raney, R. Keith

Raoult, Vincent

Rassõlkin, Anton

Rasuo, Bosko

Rasyif, Teuku Muhammad

Ravani, Bahram

Rayson, Matthew

Razmkhah, Omid

Redlarski, Grzegorz

Redutskiy, Yury

Rego, Francisco

Reguero, Borja

Reimer, James

Reja, Md Yousuf

Rem, Peter C.

Ren, Zhengru

Rezaei-Gomari, Sina

Ribeiro, Luís Frölén

Ribeiro, Tiago
Ricci, Alessio

Richmond, Robert

Rijnsdorp, Dirk

Rimoldi, Simona

Rizvi, Md Jahir

Rizzo, Fabio

Robins, Peter

Rodella, Ilaria

Rodrigues, José Miguel

Rodriguez-Perez, Daniel

Rodriguez-Zurrunero, Roberto

Rødseth, Ørnulf

Rohacs, Jozsef

Romano, Alessandro

Romero, Fernando Castaño

Rosa, Pierluigi De

Roué, Mélanie

Roy, Sukanta

Ruban, Dmitry R.

Rudan, Smiljko

Ruiz-Sinoga, José Damián

Ruju, Andrea

Ruol, Piero

Ruponen, Pekka

Russo, Vincenzo

Rusu, Eugen

Rusu, Liliana

Ružić, Igor

Sabaliauskas, Tomas

Sadat, Hamid

Saffari Pour, Mohsen

Sahu, Chunendra K.

Said, Alicia

Sakai, Atsushi

Sakamoto, Nobuaki

Salehi, Mehrdad

Salmun, Haydee

Salvetti, Ovidio

Samaras, Achilleas

Sampaio, Ana

Sampath, Dissanayake

Sánchez-Arcilla, Agustín

Sands, Timothy

Santana Rodríguez, Juan José

Santos, Angela

Santos, João Pedro

Santos, José

Santos, José Maria

Santos, Paulo

Saponieri, Alessandra

Saprykina, Yana

Sasaki, Noriyuki 
Saulnier-Talbot, Émilie

Saviano, Simona

Savvidis, Yiannis

Sawik, Bartosz

Saylam, Kutalmis

Sayol, Juan M.

Scalco, Eleonora

Scalera, Lorenzo

Scaradozzi, David

Schendel, Alexander

Schinas, Orestis

Schmidlin, Thomas

Schmitt, Pal

Schneider-Muntau, Barbara

Schoefs, Franck

Schoenrock, Kathryn M.

Schoonover, Joseph

Schuhmann, Peter

Scrano, Laura

Scully, Brandan

Scuro, Carmelo

Seiffert, Betsy

Sell, Nathan P.

Semedo, Alvaro

Senčić, Tomislav

Sendra, Sandra

Sepic, Jadranka

Sepúlveda Allende, Andrés

Serra, Roger

Sesana, Raffaella

Severino, Ricardo

Shafiee, Mahmood

Sharma, Anurag

Shcherbakova, Viktoria

Shemer, Lev

Shepard, Samuel

Sherman, D.J.

Sherwood, Christopher

Shi, Chengzhi

Shugan, Igor

Shuster, Jeremiah

Sidik, Frida

Sidoti, David

Siikonen, Timo

Silvestre, Miguel

Simarro, Gonzalo

Simonetti, Irene

Simpson, Matthew J.R.

Sinama, Frantz

Singh, Yogang

Sinha, Nityanand

Siodla, Krzysztof
Sironić, Andreja

Sivanesan, Iyyakkannu

Skřínský, Jan

Slaets, Peter

Slamovits, Claudio

Słomkiewicz, Piotr Marek

Smith, G. Jason

Smolensky, Dmitriy

Smyth J., Tim

Smyth, Thomas

Sobolev, Dmitri

Soja-Wozniak, Monika

Sokolovskiy, Mikhail A.

Solabarrieta, Lohitzune

Sonah, Humira

Song, Gwo-Shyh

Song, Shaopin

Soomro, Mahfooz

Soukissian, Takvor

Souppez, Jean-Baptiste R.G.

Sous, Damien

Souvermezoglou, Ekaterini

Spagnoli, Giovanni

Srinivasamurthy, Sharath

Stacul, Stefano

Stagonas, Dimitris

Stansby, Peter K

Steen, Sverre

Stender, Merten

Stevens, Craig L.

Straub, David

Sturm, Hendrik

Stutz, Hans Henning

$\mathrm{Su}$, Tsung-chow

Subburaj, Anitha Sarah

Suh, Kyung-Duck

Sułowicz, Maciej

Suo, Yu

Suslov, Sergey

Sutherland, James

Sutin, Alexander

Sutulo, Serge

Suzuki, Masakiyo

Svensson, Anders

Svilicic, Boris

Swain, Geoffrey

Szilas, Kristoffer

Szpilka, Christine

Taborda, Rui

Tafuni, Angelo

Tagg, Alexander

Tajima, Yoshimitsu 
Talone, Marco

Tam, Ivan CK

Tamagnone, Paolo

Tanaka, Hitoshi

Tani, Giorgio

Tankiewicz, Matylda

Tauveron, Nicolas

Taveira Pinto, Francisco

Tehrani, Faraz

Temiz, Irina

Terefenko, Paweł

Tester, Patricia

Thein, May-Win

Themelis, Nikos

Theotokatos, Gerasimos

Thornton, Daniel C.O.

Thresher, Robert

Tiseira, Andrés Omar

Tlidi, Mustapha

Toh, Tai Chong

Tomasicchio, Giuseppe

Tomlinson, Rodger

Toniolo, Horacio

Tõnisson, Hannes

Torkjazi, Mohammad

Torlapati, Jagadish

Tornabene, Francesco

Toxopeus, Serge

Tran Anh, Duong

Trenhaile, Alan

Triantafillou, Thanasis

Troncone, Antonello

Tsahalis, Demos T.

Tsanakas, Konstantinos

Tsokanos, Athanasios

Tsujimoto, Masaru

Tuan, Long Nguyen

Tuomi, Laura

Turkington, Thea

Twardowski, Michael

Tzang, Shiaw-Yih

Tziourtzioumis, Dimitrios

Tzu, Fu-Ming

Udovydchenkov, Ilya

Ueno, Michio

Ulazia, Alain

Ullmann, Clemens Vinzenz

Ullmann, Tobias

Umanzor, Schery

Umgiesser, Georg

Unru, Peter

Ustun, Taha Selim
Vad, Johanne

Vahtmäe, Ele

Vallée, Till

Valomon, Amandine

Valyrakis, Manousos

Van Den Berg, Janrik

Van Hassel, Edwin

Van Puijenbroek, Marinka

Van Vledder, G.Ph.

Van Walree, Frans

Vandeputte, Marc

Vannucchi De Camargo, Felipe

Vannucchi, Valentina

Varriale, Luisa

Vasar, Cristian

Vasconi, Mauro

Vasiljević, Goran

Vassalos, Dracos

Vaz, Nuno

Vega, Luis A.

Veitch, Brian

Veldman, Arthur E.P.

Veltri, Paolo

Verdun, Jérome

Verhagen, Henk Jan

Vicinanza, Diego

Vidhi, Rachana

Vieira, Vasco M.N.C.S.

Villa, Diego

Vinh, Nguyen Quang

Vinnem, Jan-Erik

Vinuesa, Ricardo

Viquez, Oscar A.

Vitiello, Luigi

Vižintin, Goran

Vlahopoulos, Nickolas

Vlastelica, Goran

Vossen, Robbert Van

Vujović, Igor

Vukelic, Goran

Wadman, Heidi

Wahlström, Mikael

Walstra, Dirk-Jan

Walter, Brambilla

Wang, Haibin

Wang, Taiping

Wang, Xiaoming

Wang, Xuan

Wang, Yi-Hui

Webber, Beau

Weintrit, Adam

Wellens, Peter 
Wemheuer, Bernd

Wenk, Jannis

Weslawski, Jan Marcin

Whelchel, Adam

Whitaker, Justine

White, Kristine

Wiegard, Bjarne

Wigley, Rochelle

Wiley, Robert

Windt, Christian

Włodek, Tomasz

Wong, Leslie

Wong, Tony

Woo, Dong Kook

Woodfield, Peter

Wöstemeyer, Johannes

Wu, Chensheng

Wu, Wei-Cheng

$\mathrm{Wu}$, Ying-Hsin

Xiros, Nikolaos I.

Yadav, Basant

Yahalom, Asher

Yakovis, Eugene

Yamada, Yohei

Yamamoto, Kristina

Yamazaki, Tatsuya

Yamazaki, Tetsuo

Yang, Chansu

Yang, Qiu

Yang, Ray-Yeng

Yang, Xiufeng

Yang, Yuan-Sen

Yapa, Poojitha

Yasukawa, Hironori

Yasunaga, Takeshi

Ye, Fei

Yelisetti, Subbarao

Yilmaz, Naz

Yim, Solomon

Yin, Chuntao
Yingyi, Liu

Yip, Tsz Leung

Yoon, Hyun-Doug

Yordanova, Veronika

You, Jikun

Youd, T. Leslie

Yu, Xiaochuan

Yuan, Zhiming

Yuhi, Masatoshi

Yun, Hae Keun

Zafirakou, Antigoni

Zakinyan, Arthur

Zalesny, Vladimir

Zamani Meymian, Nima

Zambrano-Martinez, Jorge Luis

Zamiri, Ali

Zamuda, Ales

Zanier, Giulia

Zaraphonitis, George

Zarzuelo, Carmen

Zaucha, Jacek

Zdankus, Tadas

Zeng, Xiangming

Zhan, Siyuan

Zhang, Jiashu

Zhang, Wenyan

Zhang, Xuyang

Zheng, Minghua

Zheng, Siming

Zhi Yung, Tay

Zhu, Huayang

Zhu, Xiaowei

Zia, Muhammad Yousuf Irfan

Ziebold, Ralf

Zis, Thalis

Zitti, Gianluca

Zodiatis, George

Zou, Lei

(C) 2020 by the authors. Submitted for possible open access publication under the terms and conditions of the Creative Commons Attribution (CC BY) license (http://creativecommons.org/licenses/by/4.0/). 